\title{
Application of combinatorial optimization of sensor electrode plate in three dimensional ECT system in real-time imaging.
}

\author{
Chen Feng $^{1 *}$, Liu Hongri ${ }^{1}$, Chen Duyun², Wang Lili \\ ${ }^{1}$ Rongcheng College, Harbin University of Science and Technology, Weihai, Shandong, PR China \\ ${ }^{2}$ School of Computer Science and Technology, Harbin University of Science and Technology, Haerbin, Heilongjiang, \\ PR China
}

\begin{abstract}
ECT technology has the advantages of simple structure, fast response, non-invasion, no pollution, and no radiation hazard and so on. Because of these advantages of ECT technology, this technology has been widely used for various applications such as flow measurement in chemical industry and petroleum; visualize the tooth surface using dental ECT, real-time medical imaging, revision total hip replacement etc. The research of ECT technology can be divided into two-dimensional ECT and three-dimensional ECT technology, and the current three-dimensional ECT technology has been widely studied.
\end{abstract}

Keywords: ETC, Performance index, Electrode combination.

Accepted on August 28, 2017

\section{Introduction}

ECT is a technique of electrical capacitance tomography. As an important branch of PT technology, and because of its noninvasive, real-time imaging, high security and low cost, it has been paid more and more attention by many researchers [1]. With the continuous improvement of people's material living standards, the detection of multiphase flow system is applied to various enterprises which are closely related to people's lives, for example, the purification process of domestic water, medical imaging, pharmaceuticals, food, and petrochemicals [2]. ECT system can be used to detect whether the content of solid in water is up to standard in the water pipeline in realtime. At the same time, with the change of multiphase flow, the main application of ECT system is to detect the content and distribution of each phase in multiphase flow [3].

The potential applications of electrical capacitance tomography are extended to pharmaceutical industries which includes two different methods such as electrical impedance tomography and electrical capacitance tomography. These techniques are mainly used in monitoring of granulation of pharmaceutical powders, dissolution testing, etc [4]. These methods provide improved accuracy and resolution of results.

Researchers are currently exploring the chances to develop portable diagnostic devices based on electrical capacitance tomography. The studies reveal that development of this technique can reduce the cost associated with brain diagnostics and neuroimaging [5]. The research of Abraham et al. has lead light to the fact that using electrical capacitance tomography for the imaging of human tissues can generate results in nanoseconds and this can be further combined with the 4D modeling also [6].

\section{State of The Art}

In the capacitance tomography system, the calculation of sensitivity is indispensable in the process of image reconstruction. In the three-dimensional ECT sensor, the finite element method is used to split the model. The number of dividing cells is usually measured in thousands of meters, which will slow down the speed of sensitivity and affect the imaging efficiency. Compared with the reconstructed image of two-dimensional capacitance tomography system, the 3D capacitance tomography system has higher axial resolution, with 12 plates, 4 plates on each layer, and a total of 3 layers of sensor structure.

\section{Methodology}

In the response surface optimization step, the approximate function must be formulated at first. Through the in-depth study of the response function and the fitting method, in the experiment, the effective test point is adopted to simulate the effective model in the simulation experiment, and the global optimal solution is obtained.

In the experiment, the fitting function is assumed to be global function, as shown in Equation 1.

$f(X)=\sum_{i=1}^{k} a_{i} \varphi_{i}(x) \rightarrow(1)$ 
It is assumed that the relationship between the measured data and the known values is linear, and so the first order function can be used, as shown in Equation 2.

$f(X)=\tilde{y}=a_{0}+a_{1} x_{1}+a_{2} x_{2}+\ldots+a_{k} x_{k} \rightarrow(2)$

When the response surface function is designed, the expression should be able to describe the real objective function and be as simple as possible. The goal of this paper is to solve the nonlinear problem, so the two order response function is used, as shown in Equation 3.

$f(X)=\tilde{y}=a_{0}+\sum_{i=1}^{k} a_{i} x_{i}+\sum_{i=1}^{k} a_{i i} x_{i}^{2}$

$+\sum_{i=1}^{k-1} \sum_{j=i+1}^{k} a_{i j} x_{i} x_{j} \rightarrow(3)$

In the actual application process, in order to reduce the workload, generally, the cross terms will be rejected, as shown in Equation 4.

$\tilde{y}=a_{0}+\sum_{i=1}^{k} a_{i} x_{i}+\sum_{i=1}^{k} a_{i i} x_{i}^{2} \rightarrow(4)$

In order to obtain the response surface model, it is usually necessary to test the significance and correlation to verify the effect of the model. In this chapter, the decision coefficient $\mathrm{R}$ is used to illustrate the fitting degree of the response surface function to the test data, and then to complete the correlation test of the model. $\mathrm{R}$ is the value of the range of $[0,1]$, if $\mathrm{R}=1$, the observation points can be found on the surface of the regression equation. The regression equation can accurately describe the change of the observed value. If the value of $R$ is closer to 1 , the fitting degree of response to the original function is better. If $\mathrm{R}=0$, the observation points are all outside the surface determined by the regression equation. The regression equation can't describe the change of the observed value.

\section{Result Analysis and Discussion}

The two order response function is determined, and then the experiment is carried out to determine the coefficient of the first order and the coefficient of the two orders. In the optimization space $\mathrm{D}$, the sample test point is selected. Because there are only five design variables in the optimization space, the orthogonal test method can be used to determine the level of the 5 factors. Then the experiment is carried out with 5 level orthogonal tables. The results are shown in Table 1.

Table 1. Orthogonal test scheme and results.

\begin{tabular}{lcccccc}
\hline Test number & $\boldsymbol{\theta}$ & $\mathbf{R}_{\mathbf{2}}-\mathbf{R}_{\mathbf{1}}$ & $\mathbf{d l}$ & $\boldsymbol{\alpha}$ & $\mathbf{R 2}$ & OPT \\
\hline 1 & 45 & 12 & 8 & 11 & 80 & 6.92611 \\
\hline 2 & 45 & 14 & 9 & 22 & 82 & 8.06540 \\
\hline 3 & 45 & 16 & 10 & 33 & 84 & 7.64522 \\
\hline 4 & 45 & 8 & 11 & 44 & 86 & 8.44581 \\
\hline
\end{tabular}

\begin{tabular}{lllllll}
\hline 5 & 45 & 10 & 12 & 55 & 88 & 7.34131 \\
\hline
\end{tabular}

In order to determine the response surface coefficient, in Table 2 , the minimum set of OPT values is selected and brought into the two order response function, and then the first and two order coefficients can be obtained. When the undetermined coefficients of the two order response function are determined, they are transformed into polynomial functions which can be solved directly. After determining the response surface model, it is usually necessary to test the reliability and analyze the error results. If the error is large, the fitting effect is poor, and the simulation of the original function is not enough, needing improvement. If the error is close to 0 , the fitting precision of the model is high and the accuracy is better. Table 2 shows the response surface error analysis and reliability test.

Table 2. Test and error value of response surface model.

\begin{tabular}{lll}
\hline Checkpoint & Error value (\%) & Error sum \\
\cline { 1 - 2 }$(45,12,8,11,80)$ & 1.05 & \\
\cline { 1 - 2 }$(45,16,10,33,84)$ & 0.28 & \\
\cline { 1 - 2 }$(45,8,11,14,86)$ & 0.09 & \\
\hline$(48,12,9,33,82,82)$ & -0.45 & 1.29
\end{tabular}

By the above table, it is found that the response surface function can be trusted. It can be concluded that by using the response surface method, extracting the sensor optimization function, establishing the two order response function test, obtaining the initial data, and carrying out the response function. The display form of response function can be obtained, and the extremum of the display function can also be obtained.

\section{Conclusions}

As a kind of process imaging technology, electrical capacitance tomography system has a very broad application prospect in the field of industrial detection because of its unique safety performance, non-invasive detection, low cost and so on. Because of its structural limitations, the two-dimensional ECT system can't truly reflect the three-dimensional distribution of each phase of the measured object. In order to improve this phenomenon, the structure of 3D ECT sensor was analyzed and optimized in this paper. And the application of combinatorial optimization in real time imaging was also studied. Based on the analysis of the traditional capacitance tomography system and the three-dimensional capacitance tomography system, the ECT sensor system, the data acquisition system and the imaging computer were studied. And the working principle of two-dimensional ECT system was briefly introduced. The use of ECT in medical imaging will have great challenges to overcome but the ongoing researches shows that the development of this technique will be able to bring revolutionary changes in the field of clinical medicine and treatment. 


\section{Acknowledgement}

1. Fund: Heilongjiang Youth Science Foundation, Name: Research on the Theory of Inverse Problem Solving and Image Fusion Method for Electrical Capacitance Tomography number: QC2012C059)

2. Fund: The National Natural Science Fund, Name: Research on Imaging of Extracting for Traditional Chinese Medicine based on Electrical Capacitance Tomography number: 60972127)

3. The National Natural Science Fund, name: Research of twophase flow regime identification and image reconstruction for Electrical Capacitance Tomography number: 60572153)

\section{References}

1. Zhao Y L, Guo B L, Yan YY. Capacitance tomography technology and the research progress of analysis. J Instrument Meters 2012; 33: 1909-1920.

2. Wang F, Marashdeh Q, Fan LS, Warsito W. Electrical capacitance volume tomography: design and applications. Sensors (Basel, Switzerland) 2010; 10: 1890-1917.

3. Yan CS, Liao YB, Tian Q. Tomography image reconstruction algorithm in. J China Optics 2013; 6: $617-632$.
4. Rimpilainen V, Heikkinen LM, Kuosmanen M, Lehikoinen A, Voutilainen A, Vauhkonen M, Ketolainen J. An electrical impedance tomography-based approach to monitor in vitro sodium chloride dissolution from pharmaceutical tablets. Rev Sci Instrum 2009; 80: 103706.

5. Wei HY, Soleimani M. Electromagnetic Tomography for Medical and Industrial Applications: Challenges and Opportunities. Proceed IEEE 2013; 101: 559-565.

6. Abraham BB, Anitha G. Designing of lab view based electrical capacitance tomography system for the imaging of bone using NI ELVIS and NI USB DAQ 6009. Bonfring Int J Power Syst Integrat Circuits 2012; 2: 1.

\section{*Correspondence to}

Chen Feng

Rongcheng College

Harbin University of Science and Technology

PR China 